\title{
Neurotoxic esterase: not so toxic?
}

\author{
James P. O'Callaghan \\ Molecular Neurotoxicology Laboratory, Toxicology and Molecular Biology Branch, Health Effects Laboratory Division, Centers for Disease Control and \\ Prevention-NIOSH, Morgantown, West Virginia 26505,USA.e-mail: jdo5@cdc.gov \\ Published online 17 March 2003; doi:10.1038/ng1135
}

An altered form of an esterase has been implicated in the development of neurotoxicity after exposure to organophosphates. Mice deficient in this enzyme should be less susceptible to toxicity, but the opposite turns out to be the case.

Although of recent concern because of their use as chemical warfare agents, organophosphates have long been used as pesticides. As a class of compounds, organophosphate esters are widely recognized for their potential to inhibit serine-containing esterases owing to phosphorylation of serine residues at the active site of these enzymes, the most notable of which is acetylcholinesterase. Most features of the acute toxicity of these compounds (used as nerve gas or as pesticides) relate to their inhibition of this enzyme.

A less well known feature of some organophosphates is their propensity to cause a delayed neuropathy that has been termed organophosphate-induced delayed neurotoxicity (OPIDN; ref. 1). OPIDN is a progressive neurological condition characterized by weakness, ataxia and subsequent paralysis of the limbs ${ }^{1,2}$. The major neuropathological hallmarks of OPIDN are degeneration of the long axons of the spinal cord and peripheral neurons, although assessments of neurotoxicity using sensitive stains for neurodegeneration show that selected regions of the brain are also involved ${ }^{3}$.

Revisiting the NTE hypothesis. Pathways leading to acute toxicity and delayed neuropathy associated with exposure to organophosphates. According to a long-standing concept in neurotoxicology, the activity of a target esterase (neurotoxic esterase or neuropathy target esterase) is inhibited by organophosphorylation at a key serine residue (refs. 4,$5 ;$ a). When inhibition of enzyme activity reaches $70-90 \%$ and, when phosphorylated NTE is modified by loss of a functional group (R), the enzyme is 'aged' and initiates the steps leading to delayed neuropathy and less prominent acute toxic effects. By generating $\mathrm{Nte}^{+/-}$mice, Winrow et al. ${ }^{7}$ showed that mice with less NTE and lower activity of NTE are more sensitive to the toxic effects of prototypical organophosphate compounds (b), findings that seem to rule out the toxic gain-of-function (aged) phenotype that serves as the key feature of the NTE hypothesis. Alternative possibilities should be considered (c) because organophosphates have the potential to phosphorylate a variety of key serinecontaining substrates, leading to altered function of a given protein ${ }^{10}$ and potentially accounting for acute and delayed neurotoxic effects.
For over 30 years, the proposed target for initiation of OPIDN has been an enzyme activity called neurotoxic esterase $^{4}$ or neuropathy target esterase (NTE; ref. 5). Despite decades of research, NTE has only recently been identified as a bona fide protein 6 . The mechanistic basis for initiation of OPIDN through NTE is largely operationally defined: the key feature of the NTE hypothesis of OPIDN involves the generation of an 'aged' form of the enzyme (ref. 5; see figure). In the accompanying paper, however, Christopher Winrow and colleagues ${ }^{7}$ provide evidence against the aging concept without ruling out involvement of NTE itself.

\section{Out with the old}

So, just what is aged NTE and why is it required for OPIDN? The aged NTE concept was conceived when it was discovered that not all organophosphates that

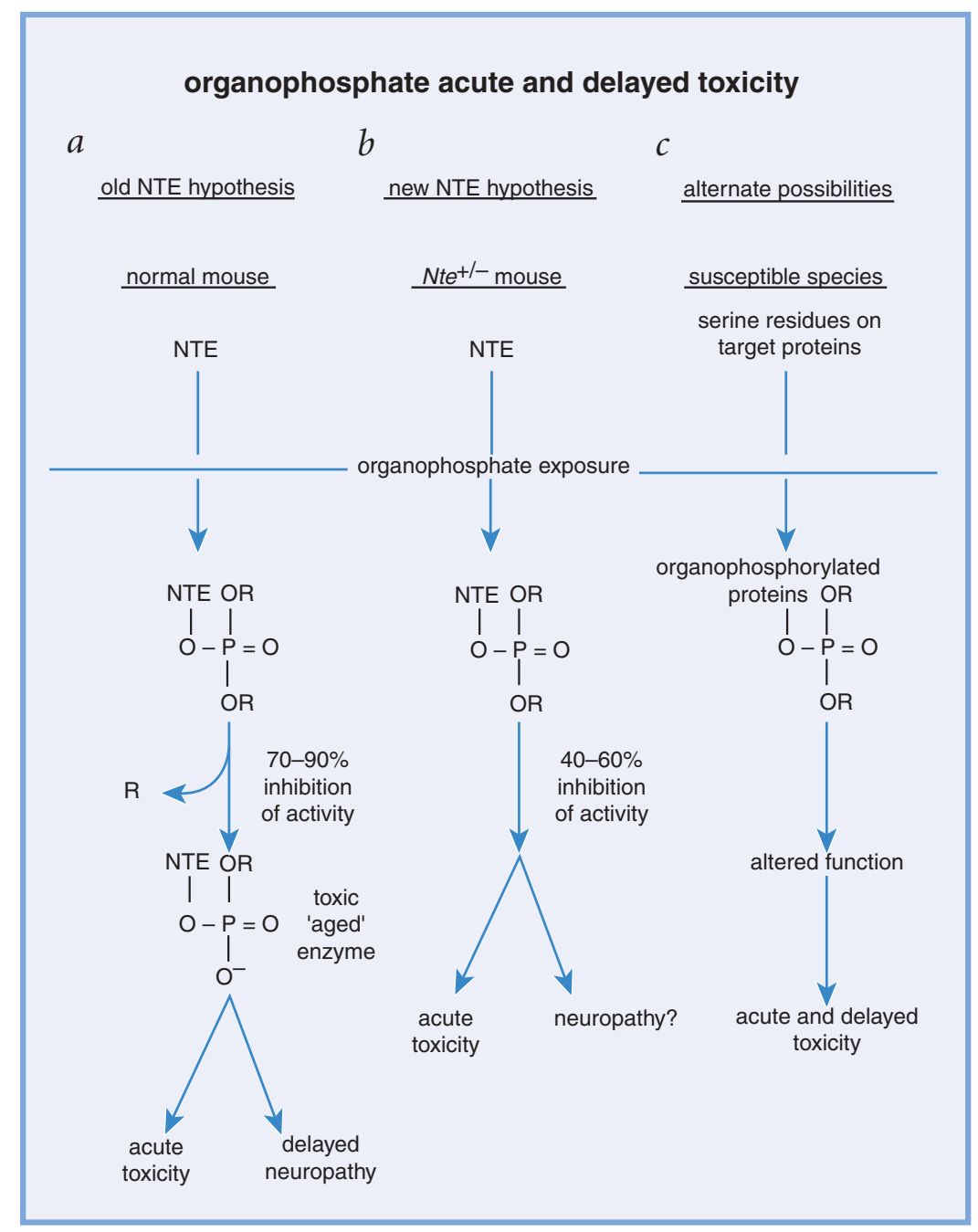


inhibited NTE activity resulted in OPIDN. Only those organophosphates that both inhibited NTE by $70-90 \%$ and modified its structure to produce a negatively charged (aged) enzyme subsequently resulted in OPIDN ${ }^{5}$. Of course, there have been exceptions to this rule, but, by and large, aged NTE, like a fine wine, represents a product that has been widely appreciated. Although they are not the first to point out potential problems with the aging NTE concept, Winrow and colleagues ${ }^{7}$ now report results that may damage this theory extensively.

They recognized that the multistep NTE hypothesis of OPIDN development might be an epiphenomenon, despite the elucidation of the structure of NTE and the assignment of some potential functions of NTE (in Drosophila melanogaster) that were linked to neurodegeneration ${ }^{6}$. They generated mice lacking NTE, which died during embryogenesis. Mice heterozygous with respect to disrupted Nte $\left(\mathrm{Nte}^{+/-}\right)$, however, survived and had lower levels of NTE protein and NTE activity but normal levels of acetylcholinesterase. Surprisingly, when the $\mathrm{Nte} \mathrm{H}^{+-}$mice were exposed to a potent organophosphate inhibitor of NTE, ethyl octylphosphonofluoridate (EOPF), the toxic effects of this compound were enhanced (see figure). Thus, lower levels and activity of NTE — and not aging or, as Winrow et al. ${ }^{7}$ so aptly describe it, a gain of function of NTE-seems to be responsible for the toxic effects of a prototypical organophosphate (EOPF) known to inhibit NTE and cause OPIDN.

\section{Neurotoxic or not?}

These findings refer to enhanced toxic effects (mainly mortality and motor activ- ity changes) rather than specifically neurotoxic (neuropathological) effects; greater susceptibility of $\mathrm{Nte}^{+/-}$mice to OPIDN was not shown. Unfortunately, the greater sensitivity of the $\mathrm{Nte}^{+/-}$mice to the toxic effects of EOPF precluded the opportunity to increase the inhibition of NTE to the $70-90 \%$ level thought to be required for initiation of OPIDN, and the mice did not survive long enough to allow neuropathological examination for the presence of OPIDN-like effects. These are disappointing shortcomings of the study, but ones that potentially can be addressed in the future by using different, less acutely toxic organophosphate analogs.

Undoubtedly, some will criticize the results simply because they were obtained in a mouse model rather than the sensitive experimental species of choice, the chicken. Such criticisms seem shortsighted, however, as this particular mouse model of OPIDN ${ }^{2}$ and others ${ }^{8}$ show a delayed neuropathological profile consistent with known cellular targets of organophosphates seen in susceptible species. The findings of Winrow et al. ${ }^{7}$ serve as a solid basis for future studies aimed at uncovering targets downstream of NTE, or in addition to NTE, that may be involved in OPIDN without the need to invoke hypothetical constructs, such as the gain-of-function aged enzyme.

In the rush to focus on NTE as a cause of OPIDN, the very real possibility that other phosphorylated proteins are important for development of acute and delayed effects of organophosphates (see figure) has been overlooked. Indeed, because protein phosphorylation represents the dominant mode of post-translational modification that underlies all neuronal function ${ }^{9}$, it would be quite surprising for the toxic (acute or delayed) effects of organophosphates to be linked to organophosphorylation of only a few substrates. Candidates abound ${ }^{10,11}$, and a few, such as key protein kinases (protein kinase A, calcium/calmodulin-dependent protein kinase II), components of the cytoskeleton and transcription factors $^{12,13}$, have recently been implicated in the development of OPIDN. As noted in previous commentaries ${ }^{14,15}$, the neurotoxicology community would be well served by further application of molecular approaches to fundamental issues in the field. Redefining the relationship between NTE and the development of OPIDN serves as but one important example of the success of such endeavors.

1. Abou-Donia, M.B. Ann. Rev. Pharmacol. Toxicol. 21, 511-548 (1981).

2. Wu, S.-Y. \& Casida, J.E. Toxicol. Appl. Pharmacol. 139, 195-202 (1996)

3. Tanaka, D. \& Bursian, S.J. Brain Res. 484, 240-256 (1989).

4. Johnson, M.K. Biochem. J. 120, 523-531 (1970).

5. Johnson, M.K. Trends Pharmacol. Sci. 8, 174-179 (1987).

6. Glynn, P. Prog. Neurobiol. 61, 61-74 (2000)

7. Winrow, C.J. et al. Nat. Genet. 33 (2003); advance online publication, 17 March 2003 doi:10.1038/ng1131.

8. Lapadula, D.M., Patton, S.E., Campbell, G.A. \& Abou-Donia, M.B. Toxicol. Appl. Pharmacol. 79, 83-90 (1985).

9. Greengard, P. Science 294, 1024-1030 (2001).

10. O'Callaghan, J.P. Neurotoxicology 15, 29-40 (1994).

11. Da Cruz e Silva, E.F. \& O'Callaghan, J.P. in Comprehensive Toxicology, Vol. 11, Nervous System and Behavioral Toxicology (eds. Sipes, G., McQueen, C.A and Gandolfi, A.J.) 181-200 (Elsevier Science, London, 1997).

12. Jensen, K.F., Lapadula, D.M., Anderson, J.K., Haykal-Coates, N. \& Abou-Donia, M.B. J. Neurosci. Res. 33, 455-460 (1992).

13. Tirupapuliyur, V.D., Abdel-Rahman, A.A., Suliman, H.B. \& Abou-Donia, M.B. Neurochem. Res. 27, 183-193 (2002).

14. Lotti, M. \& Nicotera, P. Nature 416, 481 (2002).

15. Eaton, D.L. \& Greenlee, W.F. Nature 417, 117 (2002). 